\title{
Adsorptive transport of noble gas tracers in porous media
}

\author{
Matthew J. Paul ${ }^{*}$, Steven R. Biegalski and Derek A. Haas \\ Nuclear Engineering Teaching Laboratory, The University of Texas at Austin \\ 10100 Burnet Rd, Austin, TX 78758, USA \\ "mpaul@utexas.edu \\ Justin D. Lowrey \\ Pacific Northwest National Laboratory \\ P.O. Box 999, Richland, WA 99352, USA
}

Published 10 July 2018

\begin{abstract}
The transport of noble gas radionuclides in porous media is relevant to the detection of underground nuclear detonations as well as the sequestration of reprocessing off-gases. However, in field tests releasing radioxenon underground, the quantity of radioxenon observed at the surface has fallen well below expectations. ${ }^{1}$ This study examined the diffusivity of noble gases $(\mathrm{Kr}$ and $\mathrm{Xe})$ and the inert molecular gas sulfur hexafluoride $\left(\mathrm{SF}_{6}\right)$ in porous media to observe any unexpected behavior. To replicate the transport of radiogenic noble gases in underground media, a two-bulb gaseous diffusion apparatus was constructed. The two bulbs were connected with a column of 10-30 Ottawa sand and ordinary atmosphere filled both the bulbs and pore spaces. The tracer gases were diluted in an isolated bulb to approximately $1000 \mathrm{ppm}$. Once released, the gases were allowed to diffuse through the column. Aliquots were withdrawn at regular time intervals from both bulbs and concentrations were quantified using a Shimadzu QP2010 SE gas chromatograph-mass spectrometer. The effective diffusivity was then calculated using a maximum likelihood estimate on the quasi-steady state model. The effective diffusivity of Xe in the silica sand was observed to be $135.2 \%$ that of $\mathrm{SF}_{6}$, whereas the effective diffusivity of $\mathrm{Kr}$ was observed to be $161.4 \%$ that of sulfur hexafluoride. These findings are consistent with the binary diffusivities in $\mathrm{N}_{2}: 132.6 \%$ and $161.7 \%$, respectively. However, the apparent volume of the system was inconsistent amongst the species, with Xe converging at slightly lower gas-phase concentrations than $\mathrm{Kr}$ or $\mathrm{SF}_{6}$. This apparent reduction in gas-phase concentration occurred within the first few measurements and is consistent with transient accumulation of an adsorbed phase. As the effective diffusivities in the silica sand were shown to be consistent with the binary diffusivities in $\mathrm{N}_{2}$, a porosity-tortuosity model appears to be sufficient when considering similar geological materials. However, with the observation of significant gas adsorption, consideration of adsorbed-phase accumulation is necessary when scaling to larger geological systems.
\end{abstract}

Keywords: Noble gas signatures; soil gas transport; underground nuclear explosion.

\section{Introduction}

During an underground nuclear explosion, numerous radioactive fission products will form as a direct result of fission within the event. Additionally, activation products may

This is an Open Access article published by World Scientific Publishing Company. It is distributed under the terms of the Creative Commons Attribution 4.0 (CC-BY) License. Further distribution of this work is permitted, provided the original work is properly cited. 
also form in the surrounding geological media. While many of these radioactive indicators are chemically reactive and will readily form solid compounds in the wake of the event, the noble gases are unique in their chemical inertness and gaseous state at even ambient temperatures. Consequently, radioisotopes of Xe and Ar have been identified to be of interest for detection of clandestine nuclear activities. ${ }^{1}$ However, transport processes including barometric pumping and diffusion also affect the quantity of these species detectable at the surface. While these noble gas radionuclides are of importance to underground nuclear explosion detection, relatively few field tests have been conducted using noble gases in underground transport.

In a previous study conducted by Robertson et al., $987 \mathrm{Ci}$ of ${ }^{133} \mathrm{Xe}$ was injected into basalt strata at the National Reactor Testing Station. ${ }^{2}$ While detectable quantities of Xe were detected at the surface, the quantities measured fell short of modeled expectations. More recently, a team of Pacific Northwest National Laboratory researchers injected approximately $2.5 \mathrm{Ci}$ of ${ }^{127} \mathrm{Xe}$ and $1.2 \mathrm{Ci}$ of ${ }^{37} \mathrm{Ar}$, along with sulfur hexafluoride $\left(\mathrm{SF}_{6}\right)$, into an underground cavity at the Nevada National Security Site. ${ }^{3}$ Whereas no detectible concentrations of the tracers were observed at the surface after 90 days, the cavity was then pressurized to induce advective transport of to the surface. By doing so, measurable levels of all three tracers were observed. After accounting for radioactive decay, the ${ }^{127} \mathrm{Xe}$ and ${ }^{37} \mathrm{Ar}$ appeared to be diluted by comparable factors. In contrast, $\mathrm{SF}_{6}$ was diluted by approximately one-tenth the dilution factor. As the tracers were injected simultaneously, advective transport will not lead to selective dilution. Diffusive transport of Ar may partially explain its greater dilution than $\mathrm{SF}_{6}$, but diffusion alone cannot also explain the discrepancy between $\mathrm{Xe}$ and $\mathrm{SF}_{6}$. Therefore, additional mechanisms for the dilution need to be considered.

Reviewing the literature, it is noted that primordial isotopes of Xe have been shown to be relatively scarce in the atmosphere compared to other noble gas species. ${ }^{4}$ The source of this discrepancy remains a topic of debate. However, if the same partitioning mechanism for primordial nuclides is also applicable to radionuclide transport, it would have a similarly significant impact on $\mathrm{Xe}$ transport for radioxenon monitoring applications.

One possible mechanism of separation is absorption into retained groundwater. As documented in Refs. 5-7, Xe has a relatively high solubility in water versus that of $\mathrm{Ar}$ and $\mathrm{SF}_{6}$. However, because the tests discussed in Refs. 2 and 3 were conducted in arid environments, this effect is minimized. In Ref. 8, it was demonstrated that the physicaladsorption isotherm of $\mathrm{Xe}$ is remarkably strong, enough so to propose physisorption as a significant contributor to the scarcity of primordial Xe isotopes in the atmosphere. While this missing primordial xenon question remains as an option, the existence of strong adsorption isotherms may have a significant impact on the dilution of radioxenon signatures. This work attempts to replicate the diffusive-transport process of noble gases in porous media on a laboratory scale in such a way that it isolates and identifies potential mechanisms for the observed disparity. 


\section{Methods}

The method used to examine the diffusive transport of noble gases resembles that in the work of Ney and Armistead. ${ }^{9}$ In their work, Ney and Armistead estimated the selfdiffusion coefficient of uranium hexafluoride $\left(\mathrm{UF}_{6}\right)$ by connecting two glass bulbs filled with $\mathrm{UF}_{6}$ through a capillary. The compositions of the bulbs were identical chemically, but one bulb was enriched with ${ }^{235} \mathrm{UF}_{6}$ and the other was at natural abundances. The change in isotopic abundances versus time can then be measured using a mass spectrometer. By sizing capillary and bulbs appropriately, the relaxation time of the system will be on an appropriate time scale for the mass spectrometer. Knowing the spatial dimensions of the apparatus, the self-diffusion coefficient can then be calculated from the transient concentration data.

The work performed here differs from that of Ney and Armistead in that, rather than the self-diffusion coefficient, or the binary diffusion coefficient of two gases, the objective is the effective diffusivity of the tracer materials through porous media filled with ordinary air. Because the porous media has an unknown porosity, it is not possible to directly calculate the diffusion coefficient from the transient data as was previously done. Additionally, because of sorption, the apparent volume of the system-i.e., the gasphase volume plus the equivalent volume of the sorbed gases - may not be identical for different species in the same sample.

Nevertheless, because mass spectrometry can unambiguously measure both noble gas and $\mathrm{SF}_{6}$ concentrations, it remains a viable method for estimating diffusivity. In much the same way, a porous media sample replaces the capillary as the restriction, and by sizing the sample and bulbs appropriately, the time scale of the experiment can be engineered to suit the choice of analytical instrument.

\subsection{Description of apparatus}

The apparatus consists of two bulb volumes and a bridge of 310 stainless steel piping. Porous media is held in place by stainless steel wire cloth. These are in turn held between pipe flanges and are sealed with silicone gaskets. The nominal pipe size of the system is two inches throughout the apparatus. In addition to the bulb volumes and bridge, there is an additional void space within the ball valve. A schematic diagram of the system is shown in Fig. 1. The internal volumes of the system's components were determined by water-displacement measurements. The system dimensions are listed in Table 1.

\subsection{Quantitative method}

As only low-level concentrations are observed in field-test conditions, ${ }^{2,3}$ for example parts per million for $\mathrm{SF}_{6}$ and significantly less for radionuclides, binary interactions between the tracer gases are negligible. Additionally, at low-level concentrations, saturation of adsorbent surfaces is also minimal. In lieu of noble gas radionuclides, stable noble gases were similarly desired at parts per million levels. 


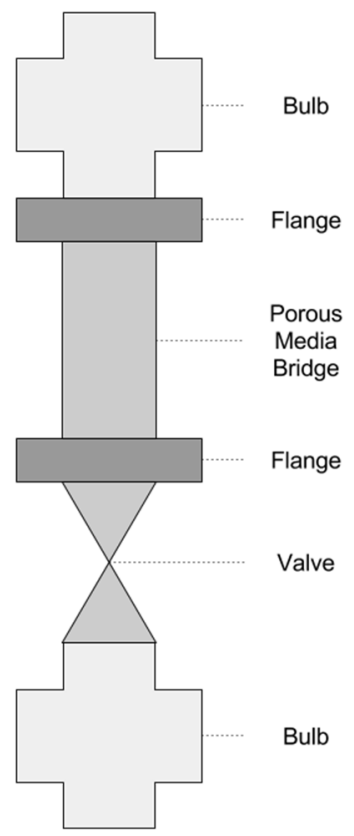

Fig. 1. Schematic diagram of the two-bulb apparatus used in this study.

Table 1. System parameters.

\begin{tabular}{cc}
\hline Parameter & Dimensions \\
\hline Length & $23.0 \mathrm{~cm}$ \\
Diameter & $5.25 \mathrm{~cm}$ \\
Volume (bulb 1) & $749 \mathrm{~cm}^{3}$ \\
Volume (valve) & $395 \mathrm{~cm}^{3}$ \\
Volume (bridge) & $498 \mathrm{~cm}^{3}$ \\
Volume (bulb 2) & $751 \mathrm{~cm}^{3}$ \\
\hline
\end{tabular}

Because of the need to measure noble gases at these low levels, a Shimadzu QP2010 SE gas chromatograph-mass spectrometer (GC-MS) was used to quantify the concentration of the tracer gases. Fifteen meters of Restek $5 \AA$ molecular sieve PLOT columns were employed to provide discernable retention time between the various gases. Helium carrier gas was carried at a constant $2.6 \mathrm{~mL} / \mathrm{min}$ through the column.

Prior to conducting a trial, the apparatus was spiked with a small quantity $(5.0 \mathrm{~mL}$ at STP) of carbon tetrafluoride $\left(\mathrm{CF}_{4}\right)$ to serve as an internal standard. Samples were withdrawn from the two-bulb apparatus using a 50- $\mu \mathrm{L}$ Hamilton Gastight syringe at regular intervals and promptly injected into the gas chromatograph with split injection.

The mass spectrometer was then operated in Selected Ion Monitoring mode using the single ionization peak of all major isotopes for the noble gases. For sulfur hexafluoride 
and carbon tetrafluoride, only the peak resulting from the removal of a single fluorine ion from the most common isotopologue was utilized (i.e., the quantitative ion mass-tocharge ratio was 127.0 for $\mathrm{SF}_{6}$ and 69.0 for $\mathrm{CF}_{4}$ ). The linearity of the response for this method was confirmed up to $2000 \mathrm{ppm}$.

\subsection{Transport theory}

The GC-MS method is limited in that the time to evaluate samples is on the order of minutes. Consequently, use of a purely transient method to evaluate diffusivity is not practical. Instead, the quasi-steady state method of Ney and Armistead (1947) was utilized.

In the two-bulb method, a restrictive bridge connects two chambers of relatively large volume. In the initial method used by Ney and Armistead (1947), this bridge was a narrow tube. For this study, the bridge was packed with unconsolidated geological media. However, so long as the characteristic relaxation time of a perturbation in the bridge is much shorter than the relaxation time of a perturbation in the bulbs, the bridge is nevertheless in a quasi-steady state. In this state, the concentration profile is essentially a linear slope bounded by the concentration in the bulbs.

To confirm that the design of this system is in quasi-steady state, the transient response within the bridge must be much faster than in the bulbs. Assuming stable tracer gases and purely diffusive transport, the material balance within the bridge must follow Eq. (1):

$$
\kappa_{i} \frac{\partial c_{i}}{\partial t}=\frac{\varepsilon}{q} \mathrm{D}_{i} \nabla^{2} c_{i}
$$

Here, $\kappa_{i}$ represents the capacity factor (also known as the retardation factor) that includes both the gas and adsorbed phase quantities; $\varepsilon$ represents the porosity; $q$ represents the tortuosity factor; and $D_{i}$ is the diffusivity in ordinary air.

Because of porosity and tortuosity effects, the diffusivity within the bridge is much smaller than that within the bulbs, which are devoid of porous media. Because of this, the concentration gradient in both bulbs is much smaller than that within the bridge. To a first approximation, the boundary conditions on the ends of the bridge are fixed concentrations. The system is axially symmetric by design and radial differences in concentration are negligible. By these assumptions, Eq. (1) can be solved by the wellknown Fourier series solution:

$$
c(z, t)=a_{0}+a_{1} z+\sum_{n=1}^{\infty} b_{n} \sin \left(\frac{n \pi}{L} z\right) e^{-\frac{n^{2} \pi^{2} \mathfrak{D}_{i}}{L^{2} \kappa_{i}} t} .
$$

From this it is clear that the slowest-moving transient eigenfunction has the following characteristic time:

$$
\tau=\frac{\kappa_{i} L^{2}}{\pi^{2} \mathrm{D}_{\mathrm{i}}}
$$




\section{J. Paul et al.}

Thus, if the characteristic response of the two-bulb system is much slower than this, the transient contributions in the bridge are negligible.

The solution to the concentration in the bulbs must be found simultaneously because the total apparent volume of the system is unknown. Assuming a linear adsorption isotherm and concentration profile in the bridge, the total quantity of tracer in the bridge is constant after the initial buildup. Consequently, at any time after the initial transient, the flux entering the low-concentration bulb must be equal to the flux leaving the highconcentration bulb. Assuming a Fickian diffusive flux, governed by the concentration at the top and bottom bulbs, this results in the coupled system of linear differential Eqs. (4):

$$
\frac{d}{d t}\left[\begin{array}{l}
C_{1} \\
C_{2}
\end{array}\right]=\mathfrak{D}_{i} \frac{A}{L}\left[\begin{array}{cc}
-\frac{1}{V_{1}} & \frac{1}{V_{1}} \\
\frac{1}{V_{2}} & -\frac{1}{V_{2}}
\end{array}\right]\left[\begin{array}{l}
C_{1} \\
C_{2}
\end{array}\right] .
$$

The solution to this system can be found by the method of eigenvalues. The first eigenvalue has a null value, which represents the equilibrium concentration. The second eigenvalue is the characteristic relaxation time of the system. Because the system does not have symmetric volumes, the eigenvector may be asymmetric. The general solution is shown in Eq. (5):

$$
\left[\begin{array}{l}
c_{1}(t) \\
c_{2}(t)
\end{array}\right]=c_{\infty}\left[\begin{array}{l}
1 \\
1
\end{array}\right]+c_{0}\left[\begin{array}{c}
-V_{2} \\
V_{1}
\end{array}\right] e^{-\mathfrak{D}_{i} \frac{A}{L}\left(\frac{1}{V_{1}}+\frac{1}{V_{2}}\right) t}=c_{\infty}\left[\begin{array}{l}
1 \\
1
\end{array}\right]+c_{0}\left[\begin{array}{c}
-V_{2} \\
V_{1}
\end{array}\right] e^{-\frac{t}{\tau}}
$$

Of primary importance is the transient eigenvalue. The inverse of this value, also known as the relaxation time, is defined in Eq. (6):

$$
\tau=\frac{1}{\mathfrak{D}_{i}} \frac{L}{A} \frac{V_{1} V_{2}}{V_{1}+V_{2}}
$$

The solution of the system has four unknowns: the equilibrium concentration, $c_{\infty}$; the effective bulb volumes, $V_{1}$ and $V_{2}$; and the relaxation time, $\tau$. The potential for a significant adsorbed volume of gas (which is chemical-species specific) precludes the use of pore volumes measured by other gases. Instead, concentrations were measured at both ends, and the apparent volume was determined as the convergence point of the two curves. A maximum-likelihood estimate was used to statistically determine the most probable value of these four parameters using data from both curves. A detailed derivation of this method is shown in Ref. 10.

\section{Results}

Tests were conducted on Ottawa silica sand sieved between \#10 and \#30 mesh. Samples were withdrawn at 10 or 15 min intervals. Three trials were conducted on the dry silica sand using $\mathrm{Kr}, \mathrm{Xe}$, and $\mathrm{SF}_{6}$ as tracer gases, and $\mathrm{CF}_{4}$ as a spiked internal standard. To remove tracer residue from previous trials, the media was flushed with compressed air for approximately 12 hours between trials. In addition, Trial 4 was conducted after first wetting the media to approximately $40 \%$ saturation. The measured concentration versus time from the first trial is shown in Fig. 2. 


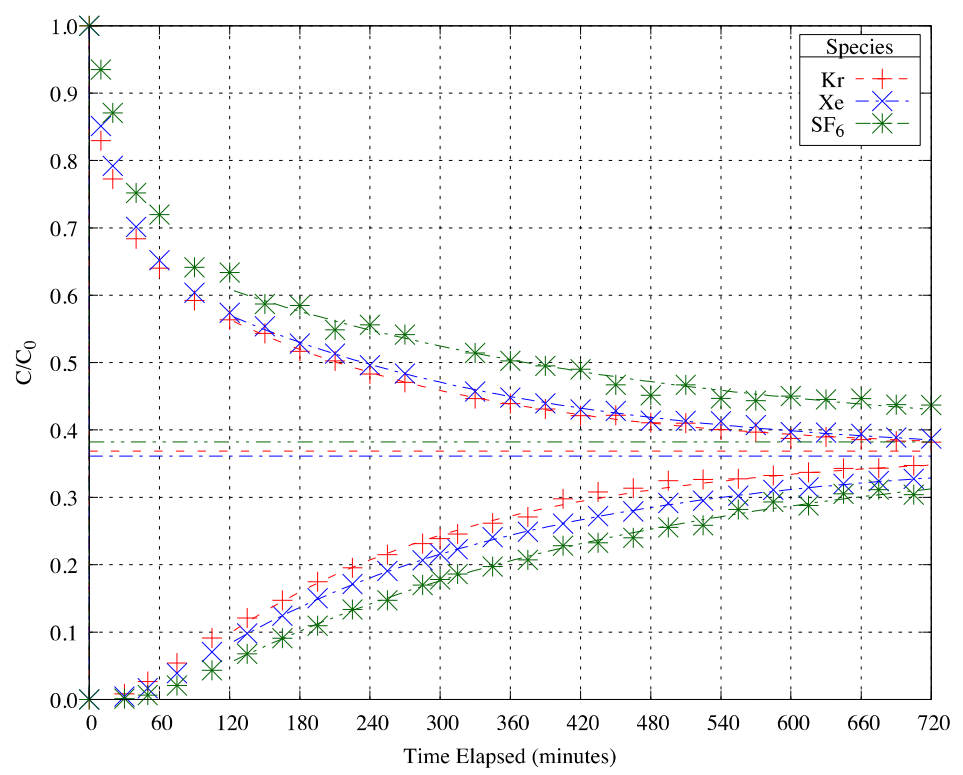

Fig. 2. Diffusivity Trial 2 (dry media) response.

The system response generally follows the theoretical exponential relaxation. The anticipated exception is at the initial buildup to a quasi-steady state. Consequently, concentrations measured in the first $120 \mathrm{~min}$ are omitted from curve fitting. In addition, the equilibrium concentration for the three species is not consistent. Xe converges at the lowest fraction, followed by $\mathrm{Kr}$ and $\mathrm{SF}_{6}$. While these differences are small, they are a function of the relative fraction of void space and geological material.

The relaxation time can be more clearly observed on the semi-logarithmic scale in Fig. 3. As the volumes of the bulbs are not identical—predominantly due to the internal volume of the ball valve - there is some separation in the y-intercept as this represents the relative magnitudes of the eigenvector. Since error was weighted on a linear scale, the curve fit more heavily weighs points far from equilibrium, and the error observed after multiple relaxation times has less bearing on the estimated slope.

For comparison, the results obtained utilizing a wet (40\% saturation) media are shown in Fig. 4. The observations are qualitatively the same, albeit with extended relaxation times for each of the three tracer gases.

The relaxation times obtained from each trial are listed in Table 2. Using the system parameters listed in Table 1, the effective diffusivities (including porosity and tortuosity) were also calculated using Eq. (6). Finally, dividing the diffusivity of the corresponding gas in $\mathrm{N}_{2}$ at $20^{\circ} \mathrm{C}$ obtained from Ref. 11 yields an estimate of the combined effect of porosity and tortuosity. As shown, these values were nearly consistent for dry media. Some deviation is possible between trials as the media considered here was 


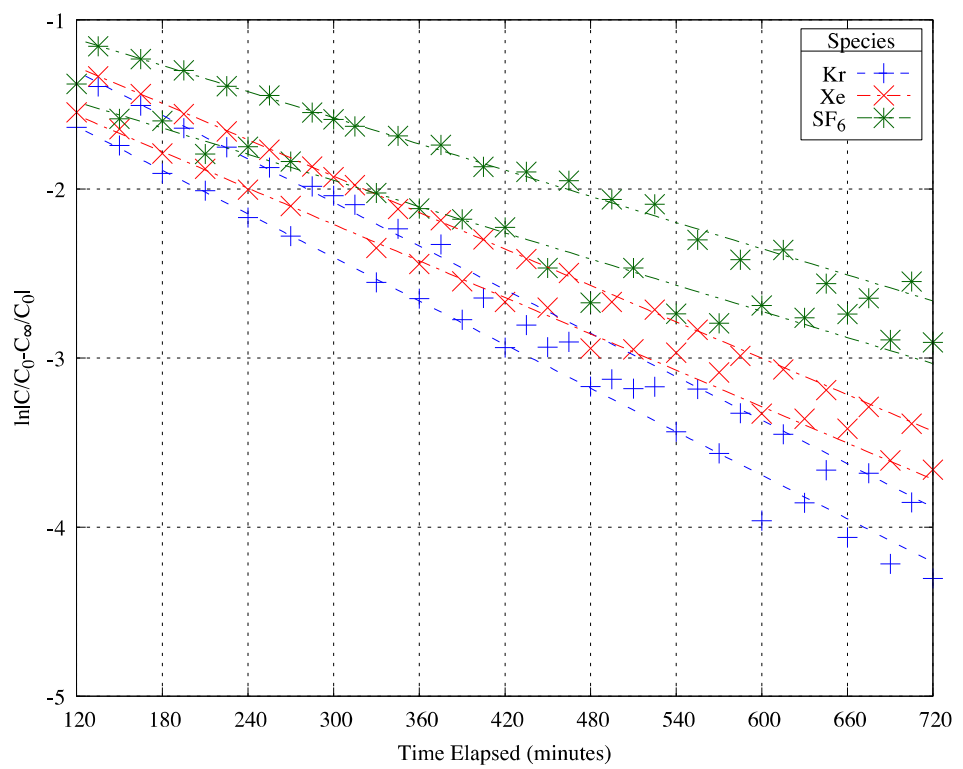

Fig. 3. Diffusivity Trial 2 (dry media) response plotted on a semi-logarithmic scale.

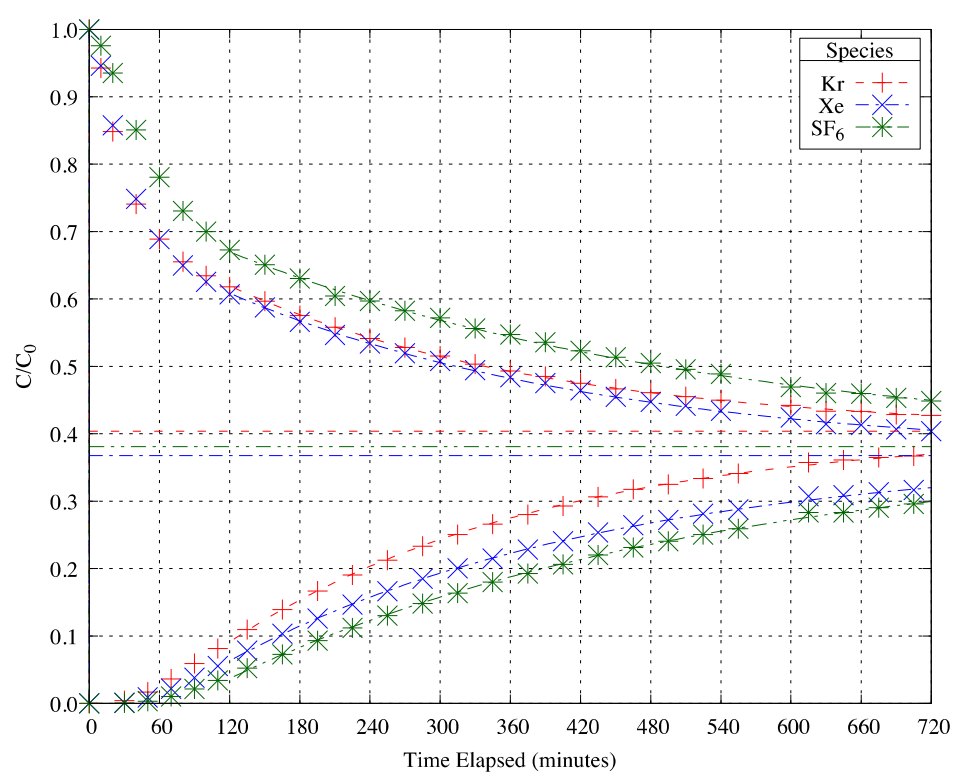

Fig. 4. Diffusivity Trial 4 (wet media) response. 
Table 2. Relaxation times of tracer gases and effective porosity-tortuosity.

\begin{tabular}{ccccc}
\hline Trial & $\mathrm{Gas}$ & $\tau($ minutes $)$ & $\mathrm{D}_{\mathrm{i}} \varepsilon / \mathrm{q}^{2}\left(\mathrm{~cm}^{2} / \mathrm{s}\right)$ & $\varepsilon / \mathrm{q}^{2}$ \\
\hline \multirow{3}{*}{1} & $\mathrm{Kr}$ & 229.6 & 0.0400 & 0.270 \\
& $\mathrm{Xe}$ & 269.2 & 0.0341 & 0.280 \\
& $\mathrm{SF}_{6}$ & 359.0 & 0.0256 & 0.279 \\
\multirow{3}{*}{2} & $\mathrm{Kr}$ & 232.9 & 0.0394 & 0.266 \\
& $\mathrm{Xe}$ & 278.6 & 0.0329 & 0.270 \\
& $\mathrm{SF}_{6}$ & 387.7 & 0.0237 & 0.258 \\
3 & $\mathrm{Kr}$ & 222.5 & 0.0413 & 0.279 \\
& $\mathrm{Xe}$ & 281.8 & 0.0326 & 0.267 \\
& $\mathrm{SF}$ & 376.7 & 0.0244 & 0.265 \\
& $\mathrm{Kr}_{6}$ & 270.5 & 0.0297 & 0.200 \\
& $\mathrm{Xe}^{\mathrm{a}}$ & 323.5 & 0.0248 & 0.204 \\
& $\mathrm{SF}_{6}$ & 416.9 & 0.0193 & 0.210 \\
\hline
\end{tabular}

Trial 4 was conducted in wet media whereas Trials $1-3$ were dry.

unconsolidated and may not settle identically if entrained when flushing the sample with compressed air.

In contrast to the relaxation times, which indicate consistent porosity-tortuosity, the observed equilibrium fractions suggest different apparent volumes. The mean equilibrium concentration as a fraction of initial concentration is listed in Table 3. Dividing by the volume of the initial bulb yields the apparent volume of the system, demonstrating a modest increase in system volume.

Table 3. Equilibrium concentration fraction.

\begin{tabular}{cccc}
\hline Trial & Gas & $\mathrm{C}_{n} / \mathrm{C}_{\Omega}$ & $\mathrm{V}(\mathrm{mL})$ \\
\hline Dry & $\mathrm{Kr}$ & 0.3650 & 2052 \\
& $\mathrm{Xe}$ & 0.3645 & 2055 \\
& $\mathrm{SF}_{6}$ & 0.3706 & 2021 \\
Wet & $\mathrm{Kr}$ & 0.4039 & 1854 \\
& $\mathrm{Xe}$ & 0.3677 & 2037 \\
& $\mathrm{SF}_{6}$ & 0.3810 & 1965 \\
\hline
\end{tabular}

The apparent volumes for $\mathrm{Kr}$ and $\mathrm{Xe}$ are approximately $30 \mathrm{~mL}$ greater than that of $\mathrm{SF}_{6}$. Spread over the mass of the sand, this indicates that adsorption of the heavy noble gases is on the order of $10^{-4} \mathrm{~cm}^{3} / \mathrm{g} /$ torr. Unfortunately, the molecular sieve column utilized in the gas chromatography is sensitive to moisture content, and the instrument sensitivity may have been altered during the course of the wet transient. Consequently, it is not clear what portion of the apparent volume difference is attributable to sorption versus changes in instrument sensitivity. 


\section{Discussion}

The method using the two-bulb gaseous diffusion apparatus demonstrated that the relative diffusivity of the tracer gas species in both wet and dry silica sand are consistent with the diffusivity of the gases in the atmosphere. Thus, while the diffusivity of Xe does exceed that of $\mathrm{SF}_{6}$, it does not do so to an extent that can fully explain the apparent disparity in concentration observed in field tests.

Despite having consistent relaxation times, significant differences are found in the apparent volume of the system in both the wet and dry cases. The increased disparity in the wet case is expected because the more soluble species has a larger apparent volume. However, the disparities in the dry case support the notion that gas adsorption may significantly influence the relative dilution of Xe. While the relative proportions of porous media and void spaces in the experiments conducted during this study diminish the magnitude of this effect, in field scenarios where the proportion of geological media versus void spaces is reversed, this effect will be enhanced. A direct measurement of noble gas adsorption on geological media is needed to provide greater clarity of this process.

\section{Acknowledgments}

This research was supported by the United Stated Department of Defense, Defense Threat Reduction Agency [HDTRA1-12-1-0009].

\section{References}

1. A. Ringbom and H. Miley, Radionuclide Monitoring, in Science for Security, ISS09, Vienna (CTBTO, 2009), p. 23.

2. J. B. Robertson, Behavior of Xenon-133 Gas after Injection Underground: Molecular Diffusion, Materials Balance, and Barometric Pressure Effects, U.S. Geological Survey OpenFile, Report IDO-22051 (1969).

3. K. B. Olsen et al., J. Radioanal. Nucl. Chem. 307, 2603 (2016).

4. H. L. Clever (ed.), Argon: IUPAC Solubility Data Series, Vol. 4 (Pergamon Press, Oxford, 1980).

5. H. L. Clever, et al., Gaseous Fluorides of Boron, Nitrogen, Sulfur, Carbon, and Silicon and Solid Xenon Fluorides in all Solvents, IUPAC-NIST Solubility Data Series 80, J. Phys. Chem. Ref. Data 34 (1), 201 (2005).

6. H. L. Clever (ed.), Krypton, Xenon and Radon: IUPAC Gas Solubility Data Series, Vol. 2 (Pergamon Press, Oxford, 1979).

7. R. O. Pepin and P. Signer, Science 149 (3681), 253 (1965).

8. F. P. Fanale and W. A. Cannon, Earth \& Planet. Sci. Lett. 11, 362 (1971).

9. E. P. Ney and F. C. Armistead, Phys. Rev. 71 (1), 14 (1947).

10. M. J. Paul, Transport and Sorption of Noble Gases in Porous Geological Media, Ph.D. dissertation, Dept. Mech. Eng., Univ. Texas, Austin, 2017.

11. T. R. Marrero and E. A. Mason, J. Phys. Chem. Ref. Data, 1 (1), 3 (1972). 